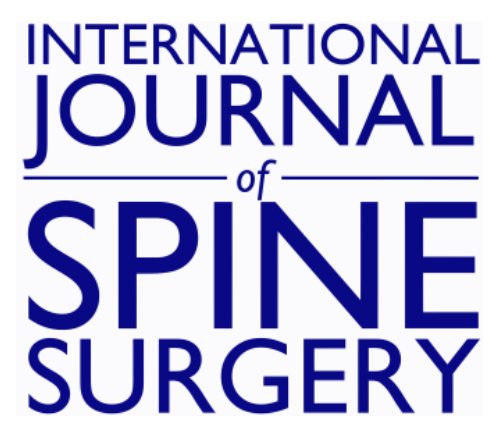

\title{
Outpatient Minimally Invasive Lumbar Fusion Using Multimodal Analgesic Management in the Ambulatory Surgery Setting
}

JAMES M. PARRISH, NATHANIEL W. JENKINS, THOMAS S. BRUNDAGE, NADIA M. HRYNEWYCZ, JEFFREY PODNAR, ASOKUMAR BUVANENDRAN and KERN SINGH

Int J Spine Surg 2020, 14 (6) 970-981

doi: https://doi.org/10.14444/7146

http://ijssurgery.com/content/14/6/970

This information is current as of April 26, 2023.

Email Alerts Receive free email-alerts when new articles cite this article. Sign up at: http://ijssurgery.com/alerts 


\title{
Outpatient Minimally Invasive Lumbar Fusion Using Multimodal Analgesic Management in the Ambulatory Surgery Setting
}

JAMES M. PARRISH, MPH, ${ }^{1}$ NATHANIEL W. JENKINS, MS,${ }^{1}$ THOMAS S. BRUNDAGE, BS, ${ }^{1}$ NADIA M. HRYNEWYCZ, BS, ${ }^{1}$ JEFFREY PODNAR, MD, ${ }^{2}$ ASOKUMAR BUVANENDRAN, MD, ${ }^{3}$ KERN SINGH, MD ${ }^{1}$

${ }^{I}$ Department of Orthopaedic Surgery, Rush University Medical Center, Chicago, Illinois ${ }^{2}$ Department of Anesthesiology, Midwest Anesthesia Partners LLC, Naperville, Illinois ${ }^{3}$ Department of Anesthesiology, Rush University Medical Center, Chicago, Illinois

\begin{abstract}
Background: The transition of minimally invasive (MIS) spine surgery from the inpatient to outpatient setting has been aided by advances in multimodal analgesic (MMA) protocols. This clinical case series of patients demonstrates the feasibility of ambulatory MIS transforaminal lumbar interbody fusion (TLIF) and lateral lumbar interbody fusion (LLIF) procedures while using an enhanced MMA protocol.

Methods: Consecutive MIS TLIF or LLIF procedures with percutaneous pedicle screw fixation and direct decompression in the ambulatory setting were reviewed. The procedures were performed using an MMA protocol. The ambulatory surgery center (ASC) did not allow for observation of patients for periods of time greater than 23 hours. We recorded patient demographics, perioperative, and postoperative characteristics.

Results: Fifty consecutive patients were identified from September 2016 to July 2019. Forty-one patients (82\%) underwent MIS TLIF, and 9 patients underwent MIS LLIF (18.0\%). All patients were discharged on the same day of surgery. The mean length of stay was 4.5 hours and 3.8 hours for the TLIF and LLIF cohorts, respectively. Our review of medical records revealed no postoperative complications following either the TLIF or the LLIF procedures.

Conclusions: The present study of 50 consecutive patients is the largest clinical series of ASC patients undergoing lumbar fusion procedures in a stand-alone facility with no extended postoperative observation capability. While using MMA protocol within the ASC, no postoperative complications were observed for either MIS TLIF or LLIF procedures. All patients were discharged from the ambulatory surgical center on the day of surgery with well-controlled postoperative pain.

Level of Evidence: 4.

Clinical Relevance: The MMA protocol is an essential aspect in transitioning minimally invasive lumbar spine surgery to the ASC. Our findings indicate that MIS lumbar fusion spine surgery with an enhanced MMA protocol can lead to safe and timely ASC discharge while minimizing hospital admission.
\end{abstract}

Minimally Invasive Surgery

Keywords: lumbar fusion, minimally invasive surgery, ambulatory surgery center, multimodal analgesia, outpatient, day surgery

\section{BACKGROUND}

An increasing number of spinal procedures are now performed in the ambulatory surgical center (ASC) setting. ${ }^{1}$ With advances in anesthetic and analgesic techniques and a trend toward minimally invasive surgical (MIS) approaches, ambulatory spine surgery is becoming more feasible. ${ }^{2}$ Furthermore, the potential for ASCs to decrease health care costs represents a financial incentive and valuebased health care. Additional benefits of outpatient surgery compared to inpatient hospitalization include decreased risk for nosocomial infections, increased safety from consistent surgical teams, and improved patient satisfaction from personalized care plans. ${ }^{3}$ While there are potential clinical and economic advantages to ambulatory spine surgery, patient safety remains an important concern, and surgeons should be aware of any possible complications, readmissions, and morbidity that can arise from outpatient procedures. ${ }^{4}$

Past investigations have examined various aspects of lumbar spine surgery performed in the ASC, such as patient selection criteria, complication rates, perioperative protocol, anesthetic techniques, and postoperative care. ${ }^{5}$ Further research is needed to 
determine whether favorable postoperative clinical outcomes can be maintained regardless of surgical setting. ${ }^{2}$ There is a scarcity of available descriptive reports regarding patients undergoing MIS lumbar fusion procedures, including transforaminal lumbar interbody fusion (TLIF) and lateral lumbar interbody fusion (LLIF). This article presents a clinical case series of patients who underwent MIS lumbar fusion procedures in the ASC with a review of literature. We focus on multimodal analgesic (MMA) protocol guidelines for the preoperative, intraoperative, and postoperative time periods. We conclude with recommendations for patient selection.

\section{METHODS}

\section{Patient Population}

This study began following institutional review board approval (ORA \#14051301). Chart review was performed for consecutive patients of a single surgeon who underwent outpatient single-level MIS TLIF or LLIF procedures with percutaneous pedicle screw fixation and direct decompression in the ambulatory setting. All patients attempted and failed nonoperative management, including antiinflammatory medications, physical therapy, and corticosteroid injections. All patients were medically cleared by their primary care physician and deemed to be in good physical health by anesthesiologists prior to surgical intervention. Patients were included if they had a diagnosis of degenerative disc disease or a grade I/II spondylolisthesis with spinal stenosis. An MMA protocol developed at our institution was used to standardize perioperative pain management for all patients (see Appendix 1). The ASC did not permit patient observation for a duration greater than 23 hours.

\section{Data Collection}

Patient demographics and baseline characteristics were recorded, including age, sex, smoking status, Charlson Comorbidity Index (CCI), and body mass index (BMI). Chronic medical conditions and comorbidities were also recorded, including complicated and uncomplicated diabetes, hypertension, asthma, arthritis, congestive heart failure, hyperlipidemia, peripheral vascular disease, malignancy, liver disease, renal failure, chronic lung disease, and gastrointestinal bleeding.
Perioperative characteristics were collected, including operative level, estimated blood loss (EBL), operative time, American Society of Anesthesiologists (ASA) score, and length of ambulatory center stay. Patient-reported visual analog scale (VAS) postoperative pain scores prior to discharge were recorded. The amount of narcotic medications administered to patients prior to discharge were converted into units of oral morphine equivalents (OME) and summed across all types of narcotic medications prescribed.

Data regarding postoperative complications were recorded, including aspiration necessitating reintubation, urinary retention requiring catheterization, urinary tract infection, acute renal failure, postoperative anemia requiring transfusion, epidural hematoma, altered mental status, venous thromboembolism, pulmonary embolism, pneumothorax, cardiac arrhythmia including atrial fibrillation and atrial flutter, ileus, pneumonia, dysphagia, atelectasis, pleural effusion, urinary manifestations of cauda equina syndrome, nausea and vomiting, and fever of unknown origin. Patient records were reviewed for any emergency room visits from the time of their lumbar spine surgery until this review was conducted in August 2019.

\section{Multimodal Analgesia Protocol}

MMA involves a combination of medications, including opioids, muscle relaxants, anticonvulsants, and anti-inflammatories (Appendix 1). The administration of pain medication can begin as early as 2-3 days before surgery. Procedure and surgeon preference may dictate beginning cyclooxygenase (COX)-2 inhibitors, such as celecoxib or meloxicam. $^{6}$ Otherwise, MMA pain control most often begins 1-2 hours before surgery. Our presurgical protocol includes orally administered long-acting opioids and muscle relaxants. ${ }^{7}$ Cyclobenzaprine is often used because it has been observed to provide lower back pain relief. ${ }^{8}$ An oral anticonvulsant, such as pregabalin, is added prior to general anesthesia. Preoperative opioid dosages are used because they are thought to induce a preemptive analgesia that ultimately lessens postoperative pain, nausea, pruritus, and the need for postoperative opioids. ${ }^{9}$ Finally, administering ketorolac can be considered during the preoperative period in order to control postoperative prostaglandin production.

Our MMA protocol calls for the use of propofol as an inhaled anesthetic, with the possible admin- 
istration of ketamine at induction. Ketamine can be used on its own or synergistically with opioids and other anti-inflammatories to reduce long-term pain and decrease postoperative opioid use.

Prior to incision, administered dexamethasone and famotidine were used to decrease the risk of nausea and vomiting. Liberal use of our standard local anesthetic injection consisting of $60 \mathrm{cc}$ Bupivacaine $0.5 \%$ with epinephrine 1:200 000 is encouraged at each surgical site. Depending on the case or surgeon discretion, several other medications can be administered for pain control and for mitigating postoperative opioid consumption. Intraoperative fentanyl $(1-2 \mathrm{mcg} / \mathrm{kg}$ in total $)$ or methadone can be given intravenously (IV). For additional pain control, IV lidocaine can be given. Prior to extubation, IV acetaminophen was used for its effect as an antipyretic and analgesic.

Management of our postoperative MMA includes using the same muscle relaxants, opioids, and anticonvulsants that were used in the preoperative setting. Cold compresses were applied to the surgical area. Surgeon discretion or case specifics dictated whether nonsteroidal anti-inflammatory drugs (NSAIDs) or acetaminophen were added. Calcium channel modulators, such as gabapentin or pregabalin, were also used on an as-needed basis. For the first 24 hours after surgery, tramadol could also be administered up to every 6 hours.

\section{Surgical Technique}

All patients underwent surgery using 1 of 2 techniques: MIS TLIF or LLIF. All MIS TLIF procedures were performed using the Wiltse technique through a paramedian $(4.5-\mathrm{cm}$ skin incision lateral to midline) approach under fluoroscopic guidance. A fascial plane was developed between the multifidus and longissimus muscles. The facet and pars were removed using a high-speed burr. Once nerve roots were identified, a laminectomy was completed. The intervertebral disc was visualized, incised, and removed. Following endplate preparation, the interbody device was placed within the intervertebral space. Finally, percutaneous pedicle screws were placed over a guide wire.

For patients undergoing LLIF, the patients were in the lateral decubitus position. A single, transverse incision was made on the lateral aspect of the body in-line. The cannulated dilator was docked on the disc center, and a guide wire was placed through the dilator. A specialized retractor was placed over the last dilator before removal. The annulus was cleared of muscle and incised. After discectomy and endplate preparation, the contralateral annulus was disrupted. The interbody cages were filled with allograft and implanted. The patient was placed in the prone position, and an 18-mm tubular retractor and guide wire were used for posterior decompression and pedicle screw placement, respectively.

\section{RESULTS}

\section{Demographic Characteristics}

A total of 50 patients were identified from September 2016 to August 2019, of which 41 patients underwent MIS TLIF and 9 patients underwent MIS LLIF. Males constituted $66.0 \%$ of all patients (Table 1). The mean age was 47.4 years for the entire cohort. Mean BMI was $27.7 \mathrm{~kg} / \mathrm{m}^{2}$. Eight patients $(16.3 \%)$ had a smoking status preoperatively. The average CCI was 0.41 .

Chronic medical conditions and comorbidities recorded for each patient were as follows: 2 patients had a history of uncomplicated diabetes mellitus, 8 patients had a preoperative diagnosis of hypertension, 1 patient had asthma, and 1 patient had hyperlipidemia. There were no patients in our study with a recorded medical history of peripheral vascular disease, myocardial infarction, malignancy, liver disease, renal failure, chronic lung disease, or gastrointestinal bleeding.

\section{Perioperative and Postoperative Outcomes}

All 50 patients underwent a single-level MIS TLIF or LLIF procedure. L4-L5 was the most common level operated on $(48.0 \%)$, followed by L5-S1 (46.0\%) (Table 2). Only the LLIF procedure was utilized to operate at the L2-L3 (11.1\%) and L3-L4 (22.2\%) levels. Mean operating time was 113.5 minutes, and mean EBL was $33.9 \mathrm{~mL}$. The mean length of stay in the surgical center following surgery was 4.4 hours. Sixteen patients had an ASA score of $1(34.0 \%)$ or $2(53.9 \%)$. There were no patients with a score of 3 or greater.

All patients in our cohort were discharged home from the surgical center on the same day as the day of surgery, with the longest postoperative stay being 8.8 hours (Table 2). The mean postoperative VAS pain score prior to discharge from the ASC was 5.1. The mean narcotics consumption following surgery before discharge was 32.4 (expressed in units of 
Table 1. Patient demographics and baseline characteristics.

\begin{tabular}{|c|c|c|c|}
\hline & $\begin{array}{c}\text { Total } \\
(\mathbf{n}=\mathbf{5 0})\end{array}$ & $\begin{array}{c}\text { TLIF } \\
(\mathrm{n}=41)\end{array}$ & $\begin{array}{c}\text { LLIF } \\
(\mathrm{n}=9)^{\mathrm{a}}\end{array}$ \\
\hline Age, mean $\pm \mathrm{SD}, \mathrm{y}$ & $47.4 \pm 8.9$ & $45.9 \pm 8.8$ & $54.2 \pm 6.4$ \\
\hline \multicolumn{4}{|l|}{ Sex, \% (n) } \\
\hline Female & $34.0(17)$ & $26.8(11)$ & $66.7(6)$ \\
\hline Male & $66.0(33)$ & $30.3(30)$ & $33.3(3)$ \\
\hline Body mass index, mean $\pm \mathrm{SD}$ & $27.7 \pm 4.4$ & $27.9 \pm 4.5$ & $26.8 \pm 3.6$ \\
\hline \multicolumn{4}{|l|}{ Smoking status, \% (n) } \\
\hline Nonsmoker & $83.7(41)$ & $87.8(36)$ & $55.6(5)$ \\
\hline Smoker & $16.3(8)$ & $9.8(4)$ & $44.4(4)$ \\
\hline $\begin{array}{l}\text { Charlson Comorbidity Index, } \\
\text { mean } \pm \text { SD }\end{array}$ & $0.41 \pm 0.55$ & $0.36 \pm 0.54$ & $0.8 \pm 0.45$ \\
\hline \multicolumn{4}{|l|}{$\begin{array}{l}\text { Ambulatory surgery center } \\
\text { score, } \%(\mathrm{n})\end{array}$} \\
\hline 1 & $34.0(16)$ & $41.0(16)$ & $0.0(0)$ \\
\hline 2 & $63.8(21)$ & $53.9(21)$ & $100(9)$ \\
\hline$\geq 3$ & $2.1(1)$ & $2.6(1)$ & $0.0(0)$ \\
\hline \multicolumn{4}{|l|}{ Preoperative diagnoses, $\%$ (n) } \\
\hline $\begin{array}{l}\text { Uncomplicated diabetes } \\
\text { mellitus }\end{array}$ & $4.1(2)$ & $4.9(2)$ & $0.0(0)$ \\
\hline Hypertension & $16.3(8)$ & $20.0(8)$ & $0(0)$ \\
\hline Asthma & $2.0(1)$ & $2.4(1)$ & $0(0)$ \\
\hline Arthritis & $4.1(2)$ & $0.0(0)$ & $22.2(2)$ \\
\hline Hyperlipidemia & $2.0(1)$ & $2.4(1)$ & $0(0)$ \\
\hline Degenerative disc disease & $72.0(36)$ & $75.6(31)$ & $55.6(5)$ \\
\hline Degenerative spondylolisthesis & $79.6(34)$ & $85.0(34)$ & $55.6(5)$ \\
\hline Isthmic spondylolisthesis & $16.3(8)$ & $20.5(8)$ & $0.0(0)$ \\
\hline Spinal stenosis & $90.0(45)$ & $90.2(37)$ & $88.9(8)$ \\
\hline Foraminal stenosis & $70.0(35)$ & $73.2(30)$ & $55.6(5)$ \\
\hline Radiculopathy & $93.9(46)$ & $95.0(38)$ & $88.9(7)$ \\
\hline
\end{tabular}

Abbreviations: TLIF, transforaminal lumbar interbody fusion; LLIF, lateral lumbar interbody fusion.

${ }^{\mathrm{a}}$ LLIF procedure involved percutaneous posterior pedicle screw instrumentation. There were no patients in our study with a recorded medical history of peripheral vascular disease, myocardial infarction, malignancy, liver disease, renal failure, chronic lung disease, or gastrointestinal bleeding.

OME). Our review of medical records revealed no postoperative complications for the patient sample (i.e., no occurrences of acute renal failure, altered mental status, aspiration, epidural hematoma, ileus, nausea and vomiting, anemia, urinary retention, urinary tract infection, or venous thromboembolism; Table 3).

\section{DISCUSSION}

\section{Patient Selection}

Careful selection of patients is important in minimizing the risk of complications. These are particularly important given the potentially limited available clinical support staff and emergency services within the ASC setting. Given the increase in outpatient spine surgery cases being performed each year, a set of best practice guidelines for selecting suitable patients may be helpful. However, to our knowledge, there are no currently standardized criteria to determine appropriate candidates for ambulatory spine surgery. ${ }^{4}$ Smith et $\mathrm{al}^{10}$ conducted a retrospective investigation focused on MIS LLIF
Table 2. Perioperative characteristics.

\begin{tabular}{|c|c|c|c|}
\hline & $\begin{array}{c}\text { Total } \\
(n=50)\end{array}$ & $\begin{array}{c}\text { TLIF } \\
(n=41)\end{array}$ & $\begin{array}{c}\text { LLIF } \\
(n=9)^{a}\end{array}$ \\
\hline \multicolumn{4}{|l|}{ Operative level, \% (n) } \\
\hline L2-L3 & $2(1)$ & $0(0)$ & $11.1(1)$ \\
\hline L3-L4 & $4(2)$ & $0(0)$ & $22.2(2)$ \\
\hline L4-L5 & $48.0(24)$ & $43.9(18)$ & $66.6(6)$ \\
\hline L5-S1 & $46.0(23)$ & $56.1(23)$ & $0(0)$ \\
\hline $\begin{array}{l}\text { Operative time, }{ }^{\mathrm{b}} \text { mean } \pm \\
\mathrm{SD}, \text { min }\end{array}$ & $113.5 \pm 27.6$ & $117.1 \pm 26.2$ & $97.8 \pm 29.7$ \\
\hline $\begin{array}{l}\text { Estimated blood loss, mean } \\
\quad \pm \mathrm{SD}, \mathrm{mL}\end{array}$ & $33.9 \pm 13.2$ & $34.5 \pm 13.6$ & $31.2 \pm 11.6$ \\
\hline $\begin{array}{l}\text { Hospital length of stay, } \\
\text { mean } \pm \mathrm{SD}, \mathrm{h}\end{array}$ & $4.4 \pm 1.6$ & $4.5 \pm 1.6$ & $3.8 \pm 1.6$ \\
\hline $\begin{array}{l}\text { Postoperative VAS pain } \\
\text { score, mean } \pm \text { SD }\end{array}$ & $5.1 \pm 2.4$ & $5.0 \pm 2.5$ & $5.8 \pm 1.5$ \\
\hline $\begin{array}{l}\text { Postoperative narcotics } \\
\text { consumption, mean } \pm \\
\text { SD, OME }\end{array}$ & $32.4 \pm 22.2$ & $31.7 \pm 21.5$ & $36.3 \pm 29.8$ \\
\hline
\end{tabular}

Abbreviations: TLIF, transforaminal lumbar interbody fusion; LLIF, lateral lumbar interbody fusion; VAS, visual analog scale; OME, oral morphine equivalents.

${ }^{a}$ LLIF procedure involved percutaneous posterior pedicle screw instrumentation. ${ }^{\mathrm{b}}$ Operative duration from surgical incision to skin closure.

and determined that factors associated with early postoperative discharge were male sex, younger age, nondeformity preoperative diagnosis, and fewer number of operative levels.

Our results are aligned with the findings of others in that the majority of patients who underwent MIS lumbar fusion in an ASC were male, were less than 50 years of age, and had been diagnosed with degenerative spinal pathology prior to undergoing single-level elective procedures. The descriptive findings we present provide a useful framework for patient selection within the lumbar fusion ambulatory setting.

Guidelines specific to lumbar fusion in the ASC have yet to be developed. However, other outpatient spine surgery recommendations, combined with the demographic characteristics of our patient population in the present study, led to the following

Table 3. Postoperative complications.

\begin{tabular}{lccc}
\hline Complication & $\begin{array}{c}\text { Total } \\
(\mathbf{n}=\mathbf{5 0 )}\end{array}$ & $\begin{array}{c}\text { TLIF } \\
(\mathbf{n}=\mathbf{4 1 )}\end{array}$ & $\begin{array}{c}\text { LLIF } \\
(\mathbf{n}=\mathbf{9})^{\mathbf{a}}\end{array}$ \\
\hline Acute renal failure & 0 & 0 & 0 \\
Altered mental status & 0 & 0 & 0 \\
Aspiration & 0 & 0 & 0 \\
Epidural hematoma & 0 & 0 & 0 \\
Ileus & 0 & 0 & 0 \\
Nausea and vomiting & 0 & 0 & 0 \\
Anemia & 0 & 0 & 0 \\
Urinary retention & 0 & 0 & 0 \\
Urinary tract infection & 0 & 0 & 0 \\
Venous thromboembolism & 0 & 0 & 0 \\
\hline
\end{tabular}

Abbreviation: LLIF, lateral lumbar interbody fusion.

${ }^{a}$ LLIF procedure involved percutaneous posterior pedicle screw instrumentation. 
exclusion criteria: age greater than 65 years; BMI greater than $40 \mathrm{~kg} / \mathrm{m}^{2}$; increased risk of postoperative nausea and vomiting; history of myocardial infarction, severe asthma, or epilepsy; and ASA score greater than or equal to 3 (Appendix 2). Perioperative characteristics that may preclude surgery in an ASC include prolonged operative duration; increased invasive nature of the procedure, such as multilevel cases; open approaches rather than MIS; and complex spinal pathologies that are nondeformity cases and may be more involved.

\section{Multimodal Analgesia Overview}

Analgesic techniques are an essential aspect of effective perioperative pain management for lumbar fusion surgery in the ASC. ${ }^{11}$ For the management of postoperative pain, numerous investigations have demonstrated the efficacy of MMA, which is the simultaneous use of multiple synergistic analgesic medications. ${ }^{1-13}$ The goal of multimodal pain management is to use a combination of therapies that act on different pathways for a local and systemic analgesic effect while reducing the dosage of any 1 medication, while the synergistic effect targets pain pathways, and the lessened medication dose minimizes the risk of individual medication side effects. For example, use of nonsteroidal antiinflammatories should be minimized in cases of spinal fusion because they can hinder arthrodesis. ${ }^{14}$ An effective protocol must include the careful selection of medications in order to reduce postoperative nausea and vomiting (PONV) as well as postoperative urinary retention (POUR). The development of these postoperative complications may hinder rapid discharge from the surgical center. A multidisciplinary team composed of orthopedic surgeons and anesthesiologists from our institution implemented a standardized MMA protocol for patients undergoing spinal fusion in an ambulatory setting (Appendix 1). ${ }^{10}$

\section{Early Preoperative Options}

As an early MMA component, COX-2 inhibitors can be used as early as 2-3 days preoperatively due to their efficacy on postoperative analgesia and due to their benefit in reducing the risk of postoperative bleeding. ${ }^{15} \mathrm{COX}-2$ inhibitors reduce prostaglandins. This is attractive because lower levels of prostaglandins can reduce hypersensitivities and also aid in a general inflammatory process reduction. Other surgical fields have noted enhanced recovery after surgery (ERAS) protocols that utilize COX-2 inhibitors to be effective in reducing postoperative morphine consumption. ${ }^{16,17}$ Patients who received COX-2 inhibitors had an early gastrointestinal return to function and shorter lengths of hospital stay. ${ }^{16}$ As discussed below, these benefits must be weighed in conjunction with the possible effect that COX inhibitors can have on bone metabolism.

\section{Drugs for Both Pre- and Postoperative Settings}

Anticonvulsants, such as gabapentin and pregabalin, are useful in the pre- and postoperative settings because of their unique mechanisms of action. During surgical trauma, voltage-gated calcium channels, such as the alpha-2 delta protein channels, are up-regulated. The channels normally allow for calcium influx followed by neurotransmitter release. Gabapentin and pregabalin exert their analgesic activity by inhibiting the alpha-2-delta protein. Given the central nervous sensitization that is induced during surgery, preoperatively slowing the calcium influx appears to reduce postoperative analgesic requirements. ${ }^{18}$ The administration of gabapentin and pregabalin after surgery has demonstrated dose-dependent effectiveness in postoperative opioid reduction. ${ }^{19}$ Dosing of pregabalin has been observed to have superior effects with the preand postoperative administration of $150 \mathrm{mg}$ versus 75 mg. ${ }^{20}$ Similarly, Gabapentin appears to have superior effects with pre- and postoperative dosings of $600 \mathrm{mg}$ versus $300 \mathrm{mg} .{ }^{21}$ While both pregabalin and gabapentin share some similarity, gabapentin is more often reported to have sedative qualities. ${ }^{22}$ Regardless, when compared to opioids, gabapentin has been associated with reductions in pruritus, nausea, and vomiting.

Ketorolac is another drug that may also be considered in the pre- and postoperative settings. As an anti-inflammatory, ketorolac functions by blocking prostaglandins. Ketorolac has also been observed to have opioid-sparing effects due to its capability to induce analgesia. ${ }^{23-25}$ While prostaglandins do have numerous roles in the upregulation of inflammatory pathways, prostaglandin inhibitors, such as Ketorolac, can also affect bone metabolism modulation. Impaired bone healing has been reported with Ketorolac doses in excess of $120-240 \mathrm{mg} / \mathrm{d}^{26,27}$ or when given for longer durations. $^{28}$ 


\section{Intraoperative Medications}

Use of ketamine during induction is attractive because it antagonizes N-methyl-D-aspartate (NMDA) receptors. These receptors activate central nervous system excitability and may have a role in opioid receptor modulation. Ketamine has been demonstrated to reduce pain levels ${ }^{29}$ and opioid consumption either by itself or when used with acetaminophen, opioids, or NSAIDs. ${ }^{30}$

Intraoperative dexamethasone has also been associated with reduction in postoperative pain scores, and when used with peripheral nerve blocks, it has been demonstrated to delay postoperative intake of analgesics. ${ }^{31}$ De Oliveira et $\mathrm{al}^{32}$ observed that, when given in excess of $0.2 \mathrm{mg} / \mathrm{kg}$, high-dose dexamethasone reduced postoperative opioid consumption. As with other opioid-sparing medications, methadone has several properties that mediate this effect. Methadone's attributes include a long half-life and inhibition of NMDA receptors as well as inhibition of serotonin and norepinephrine reuptake. Compared to patients who received IV hydromorphone, those who received postoperative methadone had improved postoperative pain scores and reduced opioid consumption. The effects may improve mood and can reduce tolerance with opioids. ${ }^{33}$ Intraoperative IV lidocaine is a part of the MMA protocol due to its ability to lower pain scores. Farag et $\mathrm{al}^{34}$ observed that when given $2 \mathrm{mg} /$ $\mathrm{kg} / \mathrm{h}$ of IV lidocaine, patients had reduced levels of pain and fewer 30-day postoperative complications. While lidocaine has not revealed statistically significant reductions in postoperative opioid consumption, it is thought that this limitation may occur due to the type of pain experienced during spine surgery. Finally, acetaminophen is beneficial just prior to extubation as an analgesic and antipyretic. Like lidocaine, IV acetaminophen has not been demonstrated to reduce opioid consumption, though it has been observed to have numerous benefits. IV acetaminophen typically delivers pain relief in 25 minutes or less and has been demonstrated to reduce extubation times, postoperative care durations, and overall lengths of stay. ${ }^{35}$ Nonetheless, the role of IV acetaminophen in ERAS is a subject of ongoing research. $^{36}$

\section{Postoperative Medications}

As a $\mu$-opioid receptor agonist, Tramadol has analgesic properties due to its inhibition of seroto- nin-norepinephrine activity. Other more potent opioids, such as hydrocodone or oxycodone, may be substituted with Tramadol. We also have incorporated ice pack use into our protocol. In at least 1 cross-sectional study on elective spine surgery, the authors observed a more positive association with cryotherapy and early rehabilitation as compared to painkiller management alone. Although 1 randomized controlled trial has demonstrated a $30 \%$ reduction in pain among women after having a spontaneous birth, this level of evidence is still needed within elective spine surgery.

\section{Regional Options for Opioid-Sparing Pain Control}

There are numerous methods to regionally anesthetize patients during spine surgery. Other methods include a single epidural injection to reduce postoperative opioid requirements. ${ }^{37}$ Another method includes establishing plane blocks on the erector spinae using continuously infusing catheters to provide anesthesia to spinal nerves. ${ }^{38}$ Plane blocks and other methods of local regional anesthetics have been observed to decrease or completely eliminate opioid consumption. ${ }^{39,40}$ Furthermore, there are numerous lumbar surgery case reports that have elaborated the sole use of either spinal or epidural anesthesia techniques during lumbar surgery. ${ }^{41,42}$

\section{Postoperative Complications and Follow-Up}

Pre- and postoperative patient counseling included education and shared decision-making techniques. Preoperatively, patients were educated about common medication side effects that might be expected and furthermore had the opportunity to discuss any psychological challenges they felt, such as preoperative fear or anxiety. The postoperative discussion included how and when patients could seek care if required. If a potentially serious complication developed, patients were informed that they would need to report to their nearest emergency department. After discharge, patients were contacted with a follow-up phone call within 1 workday. A follow-up appointment with the surgeon was scheduled in the clinic 4-6 weeks after the procedure.

\section{Postoperative Nausea and Vomiting}

One potential obstacle that can prevent same-day discharge following spinal fusion surgery is the development of PONV. ${ }^{43}$ Patients with past nausea 
or vomiting after surgical procedures or those at higher risk for developing PONV can be screened with the transdermal application of a scopolamine patch preoperatively. Although maintenance of anesthesia is often accomplished with Sevoflurane and Fentanyl, care must be taken to administer the minimum dose necessary $(1-2 \mathrm{mg} / \mathrm{kg})$ in order to induce a clinical effect. This may be a reason why we did not observe an association with postoperative visual loss. Another significant contributor to PONV is the use of opioid medications. To prevent this, local anesthetics are applied intraoperatively, and nonnarcotic medications are used to reduce the development of PONV. ${ }^{43}$ Adequate hydration and preoperative administration of antiemetics, such as ondansetron or metoclopramide for refractory symptoms, are also recommended strategies for limiting PONV. ${ }^{44}$

\section{Postoperative Urinary Retention}

POUR following lumbar fusion procedures is another possible barrier to same-day discharge following surgery. POUR can be defined as a bladder scan greater than $300 \mathrm{~mL}$, the postoperative necessity of a straight catheterization, or a urology consultation. Previous research has demonstrated that intraoperative usage of phenylephrine and neostigmine may increase the risk of developing POUR. ${ }^{45}$ Although isolated cases of POUR may resolve without serious clinical consequences, surgeons should be mindful of this complication arising in lumbar fusion procedures as a sequelae of compressive epidural hematoma resulting in cauda equina syndrome. The rate of POUR has been determined to be as high as $20 \%$ following singlelevel MIS lumbar fusion procedures. ${ }^{45}$ As increasing lumbar fusion surgeries are being performed in the ambulatory setting, essential considerations to keep in mind include the identification of risk factors that can prognosticate the development of postoperative POUR and avoiding the use of medications that may increase this risk.

\section{Postoperative Education and Shared Decision Making}

A lack of information regarding either unknown or unexpected complications is occasionally reported as a cause of patient fear and anxiety in the outpatient surgical setting. ${ }^{46,47}$ As York et $\mathrm{al}^{48}$ report, ensuring that patient education includes topics such as normalizing common postoperative symptoms, informing patients to monitor postoperative complications, providing wound care instructions, and sharing relevant contact numbers. Furthermore, as others have observed, we engaged patients beyond education into a shared decisionmaking paradigm. ${ }^{49}$ Developing a plan with the patient may include specific actionable items. For example, patients may need to actively monitor themselves for specific symptoms. For instance, discussing situations that should prompt further health care might be noticing the development of extremity weakness, involuntary urination, or loss of stool. Having patients be aware and ready to make decisions based on their experience not only improves safety but has been observed to increase satisfaction among outpatient surgery centers. ${ }^{50,51}$

\section{Postoperative Monitoring Prior to Discharge}

There is currently no consensus on the acceptable amount of time that patients should remain in the surgical center for monitoring before discharge. The patients in our study remained in the ASC for an average of 4.4 hours after their MIS TLIF or LLIF procedure, with some patients leaving as early as 2 hours after their procedure and others leaving as late as 8 hours postoperatively. Prior to discharge, it is recommended that patients be able to tolerate oral intake, spontaneously void, have their pain adequately controlled, and pass a neurological exam. Patients in our study reported an average VAS pain score of 5.1 prior to discharge. While 1 patient did experience urinary retention, the patient recovered and was able to be discharged in just over 6 hours. Previous research has found that with adequate planning and close monitoring, these goals may be achieved hours after surgery, ${ }^{44}$ which was the case for all patients in our study. In the postdischarge setting, the role of patient education and shared decision making were paramount. Both processes were critical in facilitating patient knowledge regarding when and how it was most appropriate to seek postdischarge care. After discharge, patients were contacted with a follow-up phone call within 1 workday. A follow-up clinic appointment was scheduled within the subsequent 4-6 weeks.

\section{MIS TLIF}

The MIS TLIF procedure has demonstrated decreased intraoperative blood loss, reduced narcotics requirements, and shorter hospital lengths of 
stay compared to the traditional open approach. ${ }^{52}$ These advantages associated with the MIS approach have made the adoption of this procedure in the ambulatory setting more feasible. ${ }^{53,54}$

There are several technical facets that must be addressed when performing MIS TLIF, such as achieving appropriate decompression, endplate preparation, and instrumentation. The limited visualization and soft tissue dissection associated with MIS TLIF can make achieving sufficient decompression a challenge. If a decompression procedure was performed at the same operative level, scar formation and dural adhesions may occur, which can increase the risk of intraoperative dural tear. This complication may not only negatively impact postoperative recovery but also hinder same-day discharge.

Violation of the anterior aspect of the disc space may result in damage to the great vessels, such as the aorta, which presents a rare but devastating complication that can further inhibit the transition to the ambulatory setting. ${ }^{55}$ The minimization of nerve root and thecal retraction intraoperatively during TLIF may also help the postoperative recovery course. While mastering these technical challenges of MIS TLIF is not an easy accomplishment, prior series of patient cases regarding this procedure in the ambulatory setting have demonstrated its safety and efficacy. ${ }^{10}$

\section{MIS LLIF}

There are limited clinical data on the safety and efficacy of MIS LLIF being performed in an ambulatory setting. Patient selection criteria and sufficient experience of the surgeon may help to prevent complications. ${ }^{52,56}$ The MIS LLIF procedure allows for limited soft tissue dissection and a more thorough endplate preparation that results in a larger surface area for fusion. This procedure has been increasingly performed in recent years, with studies demonstrating a shorter recovery time and decreased blood loss. ${ }^{57}$ A preoperative MRI is often used to visualize the patient's disc space relative to their retroperitoneal anatomy. Important landmarks include the psoas muscle, the lumbar plexus, and great vessels. A more anterior positioning of the psoas muscle may place the lumbar plexus at risk while accessing the disc space from the lateral approach. Intraoperative neuromonitoring may be recommended in such cases. A lateral positioning of the great vessels may also put them at risk during the lateral approach to the disc space. ${ }^{58-60} \mathrm{An}$ intraoperative vascular injury in an ASC setting is rare but could be fatal. This emphasizes the need to evaluate regional vascular anatomy on preoperative imaging studies.

LLIF complication rates are quite varied across different studies. ${ }^{61}$ This is likely attributable to the variation in surgeon experience, patient characteristics, and utilization of different instrumentation and access systems. ${ }^{62}$ An overall $36 \%$ rate of early neurologic complications was found, which included any postoperative weakness or loss of sensation. The sensory and motor changes noted were mostly transient, and the rate of persistent neurologic complications lasting beyond 6 months was below $4 \%$ across all studies. The resolution of these symptoms within 6 postoperative months suggests that the complications were due to transient stretching of the lumbar plexus or trauma to the psoas muscle. All other complications, such as cardiac, pulmonary, wound, and vascular complications, were found to be less than $2 \%$.

\section{CONCLUSIONS}

This article presents a case series of 50 consecutive patients who underwent outpatient lumbar fusion procedures in a stand-alone ambulatory surgery setting. To our knowledge, this is the largest clinical series to date. Patients were generally healthy, with a mean age of less than 50 years, mean BMI less than 30, and low comorbidity burden with few chronic medical conditions. All procedures performed were single-level MIS TLIF or LLIF, and the mean length of stay in the ambulatory surgical center following surgery was approximately 4 hours. All patients were discharged the same day as the surgery. There were no postoperative complications that significantly delayed their discharge. A summary of guidelines and recommendations regarding patient selection and perioperative management from a review of the literature is also included in this article.

\section{REFERENCES}

1. Mundell BF, Gates MJ, Kerezoudis P, et al. Does patient selection account for the perceived cost savings in outpatient spine surgery? A meta-analysis of current evidence and analysis from an administrative database: presented at the 2018 AANS/ CNS Joint Section on Disorders of the Spine and Peripheral Nerves. J Neurosurg Spine. 2018;1(aop):1-9. https://thejns.org/ 
spine/view/journals/j-neurosurg-spine/aop/article-10.31712018.4.SPINE1864.xml

2. Sivaganesan A, Hirsch B, Phillips FM, McGirt MJ. Spine surgery in the ambulatory surgery center setting: value-based advancement or safety liability? Neurosurgery. 2018;83(2):159165. doi:10.1093/neuros/nyy057

3. Hollenbeck BK, Hollingsworth JM, Dunn RL, Zaojun Ye, Birkmeyer JD. Ambulatory surgery center market share and rates of outpatient surgery in the elderly. Surg Innov. 2010;17(4):340-345. doi:10.1177/1553350610377211

4. Ahn J, Bohl DD, Tabaraee E, Basques BA, Singh K. Current trends in outpatient spine surgery. Clin Spine Surg. 2016;29(9):384-386. doi:10.1097/BSD.0000000000000417

5. Soegaard R, Christensen FB, Christiansen T, Bünger C. Costs and effects in lumbar spinal fusion. A follow-up study in 136 consecutive patients with chronic low back pain. Eur Spine J. 2007;16(5):657-668. doi:10.1007/s00586-006-0179-8

6. Garcia RM, Cassinelli EH, Messerschmitt PJ, Furey CG, Bohlman HH. A multimodal approach for postoperative pain management after lumbar decompression surgery: a prospective, randomized study. J Spinal Disord Tech. 2013;26(6):291297. doi:10.1097/BSD.0b013e318246b0a6

7. Bohl DD, Louie PK, Shah N, et al. Multimodal versus patient-controlled analgesia after an anterior cervical decompression and fusion. Spine. 2016;41(12):994-998. doi:10.1097/ BRS.0000000000001380

8. van Tulder M, On behalf of the COST B13 Working Group on Guidelines for the Management of Acute Low Back Pain in Primary Care, Becker A, et al. Chapter 3 European guidelines for the management of acute nonspecific low back pain in primary care. Eur Spine J. 2006;15(suppl 2):s169-s191. doi:10.1007/s00586-006-1071-2

9. Bhatia A, Buvanendran A. Anesthesia and postoperative pain control-multimodal anesthesia protocol. J Spine Surg. 2019;5(suppl 2):S160-S165. doi:10.21037/jss.2019.09.33

10. Smith WD, Wohns RNW, Christian G, Rodgers EJ, Rodgers WB. Outpatient minimally invasive lumbar interbody: fusion predictive factors and clinical results. Spine. 2016;41(suppl 8):S106-S122. doi:10.1097/BRS. 0000000000001479

11. Devin CJ, McGirt MJ. Best evidence in multimodal pain management in spine surgery and means of assessing postoperative pain and functional outcomes. $J$ Clin Neurosci. 2015;22(6):930-938. doi:10.1016/j.jocn.2015.01.003

12. Dunn LK, Durieux ME, Nemergut EC. Non-opioid analgesics: novel approaches to perioperative analgesia for major spine surgery. Best Pract Res Clin Anaesthesiol. 2016;30(1):79-89. doi:10.1016/j.bpa.2015.11.002

13. Singh K, Bohl DD, Ahn J, et al. Multimodal analgesia versus intravenous patient-controlled analgesia for minimally invasive transforaminal lumbar interbody fusion procedures. Spine. 2017;42(15):1145-1150. doi:10.1097/BRS. 0000000000001992

14. Thaller J, Walker M, Kline AJ, Anderson DG. The effect of nonsteroidal anti-inflammatory agents on spinal fusion. Orthopedics. 2005;28(3):299-303; quiz 304-305. https://www. ncbi.nlm.nih.gov/pubmed/15790089

15. Elia N, Lysakowski C, Tramèr MR. Does multimodal analgesia with acetaminophen, nonsteroidal antiinflammatory drugs, or selective cyclooxygenase- 2 inhibitors and patientcontrolled analgesia morphine offer advantages over morphine alone? Anesthesiology. 2005;103(6):1296-1304. doi:10.1097/ 00000542-200512000-00025

16. Lohsiriwat V. Opioid-sparing effect of selective cyclooxygenase-2 inhibitors on surgical outcomes after open colorectal surgery within an enhanced recovery after surgery protocol. Clinical Nutrition ESPEN. 2016;12:e59. doi:10.1016/j.clnesp. 2016.02.092

17. Laine L, Curtis SP, Langman M, et al. Lower gastrointestinal events in a double-blind trial of the cyclooxygenase- 2 selective inhibitor etoricoxib and the traditional nonsteroidal anti-inflammatory drug diclofenac. Gastroenterology. 2008;135(5):1517-1525. doi:10.1053/j.gastro.2008.07.067

18. Tiippana EM, Hamunen K, Kontinen VK, Kalso E. Do surgical patients benefit from perioperative gabapentin/pregabalin? A systematic review of efficacy and safety. Anesth Analg. 2007;104(6):1545-1556. doi:10.1213/01.ane.0000261517.27532. 80

19. Schmidt PC, Ruchinelli G, Mackey SC, Carroll IR. Perioperative gabapentinoids. Surv Anesthesiol. 2014;58(2):9697. doi:10.1097/01.sa.0000443883.75625.7a

20. Kim JC, Choi YS, Kim KN, Shim JK, Lee JY, Kwak YL. Effective dose of peri-operative oral pregabalin as an adjunct to multimodal analgesic regimen in lumbar spinal fusion surgery. Spine. 2011;36(6):428-433. doi:10.1097/BRS. 0b013e3181d26708

21. Pandey CK, Navkar DV, Giri PJ, et al. Evaluation of the optimal preemptive dose of gabapentin for postoperative pain relief after lumbar diskectomy: a randomized, double-blind, placebo-controlled study. J Neurosurg Anesthesiol. 2005;17(2):65. doi:10.1097/01.ana.0000151407.62650.51

22. Ho K-Y, Gan TJ, Habib AS. Gabapentin and postoperative pain - a systematic review of randomized controlled trials. Pain. 2006;126(1-3):91-101. doi:10.1016/j.pain. 2006.06.018

23. Turner DM, Warson JS, Wirt TC, Scalley RD, Cochran RS, Miller KJ. The use of ketorolac in lumbar spine surgery: a cost-benefit analysis. J Spinal Disord. 1995;8(3):206-212. doi:10. 1097/00002517-199506000-00005

24. Le Roux PD, Samudrala S. Postoperative pain after lumbar disc surgery: a comparison between parenteral ketorolac and narcotics. Acta Neurochir. 1999;141(3):261-267. doi: $10.1007 / \mathrm{s} 007010050296$

25. Gwirtz KH, Kim HC, Nagy DJ, et al. Intravenous ketorolac and subarachnoid opioid analgesia in the management of acute postoperative pain. Reg Anesth. 1995;20(5):395401. https://www.ncbi.nlm.nih.gov/pubmed/8519716.

26. Reuben SS, Ablett D, Kaye R. High dose nonsteroidal anti-inflammatory drugs compromise spinal fusion. Can $J$ Anaesth. 2005;52(5):506-512. doi:10.1007/BF03016531

27. Li J, Ajiboye RM, Orden MH, Sharma A, Drysch A, Pourtaheri S. The effect of ketorolac on thoracolumbar posterolateral fusion: a systematic review and meta-analysis. Clin Spine Surg. 2018;31(2):65-72. doi:10.1097/BSD. 0000000000000613

28. Li Q, Zhang Z, Cai Z. High-dose ketorolac affects adult spinal fusion: a meta-analysis of the effect of perioperative nonsteroidal anti-inflammatory drugs on spinal fusion. Spine. 2011;36(7):E461-E468. doi:10.1097/BRS.0b013e3181dfd163

29. Young A, Buvanendran A. Recent advances in multimodal analgesia. Anesthesiol Clin. 2012;30(1):91-100. doi:10.1016/j.anclin.2011.12.002 
30. Loftus RW, Yeager MP, Clark JA, et al. Intraoperative ketamine reduces perioperative opiate consumption in opiatedependent patients with chronic back pain undergoing back surgery. Anesthesiology. 2010;113(3):639-646. doi:10.1097/ ALN.0b013e3181e90914

31. Clement J-C, Besch G, Puyraveau M, et al. Clinical effectiveness of single dose of intravenous dexamethasone on the duration of ropivacaine axillary brachial plexus block: the randomized placebo-controlled ADEXA trial. Reg Anesth Pain Med. 2019;44(3):e100035. doi:10.1136/rapm-2018-100035

32. De Oliveira GS, Almeida MD, Benzon HT, McCarthy RJ. Perioperative single dose systemic dexamethasone for postoperative pain: a meta-analysis of randomized controlled trials. Anesthesiology. 2011;115(3):575-588. doi:10.1097/ALN. 0b013e31822a $24 \mathrm{c} 2$

33. Murphy GS, Szokol JW, Avram MJ, et al. Clinical effectiveness and safety of intraoperative methadone in patients undergoing posterior spinal fusion surgery: a randomized, double-blinded, controlled trial. Anesthesiology. 2017;126(5):822-833. http://anesthesiology.pubs.asahq.org/arti cle.aspx?articleid=2620031

34. Farag E, Ghobrial M, Sessler DI, et al. Effect of perioperative intravenous lidocaine administration on pain, opioid consumption, and quality of life after complex spine surgery. Anesthesiology. 2013;119(4):932-940. doi:10.1097/ ALN.0b013e318297d4a5

35. Hansen RN, Pham AT, Böing EA, Lovelace B, Wan GJ, Miller TE. Comparative analysis of length of stay, hospitalization costs, opioid use, and discharge status among spine surgery patients with postoperative pain management including intravenous versus oral acetaminophen. Curr Med Res Opin. 2017;33(5):943-948. doi:10.1080/03007995.2017.1297702

36. Mörwald EE, Poeran J, Zubizarreta N, Cozowicz C, Mazumdar M, Memtsoudis SG. Intravenous acetaminophen does not reduce inpatient opioid prescription or opioid-related adverse events among patients undergoing spine surgery. Anesthes Analg. 2018;127(5):1221-1228. doi:10.1213/ane. 0000000000003344

37. Thepsoparn M, Sereeyotin J, Pannangpetch P. Effects of combined lower thoracic epidural/general anesthesia on pain control in patients undergoing elective lumbar spine surgery. Spine. 2018;43(20):1381-1385. doi:10.1097/brs. 0000000000002662

38. Chin KJ, Lewis S. Opioid-free analgesia for posterior spinal fusion surgery using erector spinae plane (ESP) blocks in a multimodal anesthetic regimen. Spine. 2019;44(6):E379-E383. doi:10.1097/BRS.0000000000002855

39. Melvin JP, Schrot RJ, Chu GM, Chin KJ. Low thoracic erector spinae plane block for perioperative analgesia in lumbosacral spine surgery: a case series. Can $J$ Anaesth. 2018;65(9):1057-1065. doi:10.1007/s12630-018-1145-8

40. Reynolds RAK, Legakis JE, Tweedie J, et al. Postoperative pain management after spinal fusion surgery: an analysis of the efficacy of continuous infusion of local anesthetics. Global Spine J. 2013;3(1):7-14. doi:10.1055/s-0033-1337119

41. Yilmaz C, Buyrukcu SO, Cansever T, Gulsen S, Altinors N, Caner H. Lumbar microdiscectomy with spinal anesthesia: comparison of prone and knee-chest positions in means of hemodynamic and respiratory function. Spine. 2010;35(11):1176-1184. doi:10.1097/BRS.0b013e3181be5866

42. Ezhevskaya AA, Mlyavykh SG, Anderson DG. Effects of continuous epidural anesthesia and postoperative epidural analgesia on pain management and stress response in patients undergoing major spinal surgery. Spine. 2013;38(15):1324-1330. doi:10.1097/BRS.0b013e318290ff26

43. Buvanendran A, Thillainathan V. Preoperative and postoperative anesthetic and analgesic techniques for minimally invasive surgery of the spine. Spine. 2010;35(suppl 26):S274 S280. doi:10.1097/BRS.0b013e31820240f8

44. Mohandas A, Summa C, Worthington WB, et al. Best practices for outpatient anterior cervical surgery: results from a delphi panel. Spine. 2017;42(11):E648-E659. doi:10.1097/BRS. 0000000000001925

45. Mayo BC, Louie PK, Bohl DD, et al. Effects of intraoperative anesthetic medications on postoperative urinary retention after single-level lumbar fusion. Spine. 2016;41(18):1441-1446. doi:10.1097/brs.0000000000001554

46. Strøm J, Bjerrum MB, Nielsen CV, et al. Anxiety and depression in spine surgery - a systematic integrative review. Spine J. 2018;18(7):1272-1285. doi:10.1016/j.spinee.2018.03.017

47. Alacadag M, Cilingir D. Presurgery anxiety and day surgery patients' need for information. J Perianesth Nurs. 2018;33(5):658-668. doi:10.1016/j.jopan.2017.06.125

48. York PJ, Gang CH, Qureshi SA. Patient education in an ambulatory surgical center setting. J Spine Surg. 2019;5(suppl 2):S206-S211. doi:10.21037/jss.2019.04.07

49. Jaensson M, Dahlberg K, Nilsson U. Factors influencing day surgery patients' quality of postoperative recovery and satisfaction with recovery: a narrative review. Perioperative Med. 2019;8(1). doi:10.1186/s13741-019-0115-1

50. Sepucha KR, Atlas SJ, Chang Y, et al. Informed, patient-centered decisions associated with better health outcomes in orthopedics: prospective cohort study. Med Decis Mak. 2018;38(8):1018-1026. doi:10.1177/0272989X18801308

51. Jaensson M, Dahlberg K, Eriksson M, Grönlund Å, Nilsson U. The development of the Recovery Assessments by Phone Points (RAPP): a mobile phone app for postoperative recovery monitoring and assessment. JMIR mHealth uHealth. 2015;3(3):e86. doi:10.2196/mhealth.4649

52. Bovonratwet P, Ottesen TD, Gala RJ, et al. Outpatient elective posterior lumbar fusions appear to be safely considered for appropriately selected patients. Spine J. 2018;18(7):11881196. doi:10.1016/j.spinee.2017.11.011

53. Eckman WW, Hester L, McMillen M. Same-day discharge after minimally invasive transforaminal lumbar interbody fusion: a series of 808 cases. Clin Orthop Relat Res. 2014;472(6):1806-1812. doi:10.1007/s11999-013-3366-Z

54. Villavicencio AT, Nelson EL, Mason A, Rajpal S, Burneikiene S. Preliminary results on feasibility of outpatient instrumented transforaminal lumbar interbody fusion. $J$ Spinal Disord Tech. 2013;26(6):298-304. doi:10.1097/BSD. 0b013e318246aea2

55. Bae HJ, Cho TG, Kim CH, Lee HK, Moon JG, Choi JI. Aortic injury during transforaminal lumbar interbody fusion. Korean J Spine. 2017;14(3):118-120. doi:10.14245/kjs.2017.14.3. 118

56. Chin KR, Pencle FJR, Coombs AV, et al. Lateral lumbar interbody fusion in ambulatory surgery centers: patient selection and outcome measures compared with an inhospital cohort. Spine. 2016;41(8):686-692. doi:10.1097/BRS. 0000000000001285

57. Ozgur BM, Aryan HE, Pimenta L, Taylor WR. Extreme 
lateral interbody fusion (XLIF): a novel surgical technique for anterior lumbar interbody fusion. Spine J. 2006;6(4):435-443. doi:10.1016/j.spinee.2005.08.012

58. Mai HT, Schneider AD, Alvarez AP, et al. Anatomic considerations in the lateral transpsoas interbody fusion. Clin Spine Surg. 2019;32(5):215-221. doi:10.1097/bsd. 0000000000000760

59. Berjano P, Lamartina C. Minimally invasive lateral transpsoas approach with advanced neurophysiologic monitoring for lumbar interbody fusion. Eur Spine J. 2011;20(9):15841586. doi:10.1007/s00586-011-1997-x

60. Campbell PG, Nunley PD, Cavanaugh D, et al. Shortterm outcomes of lateral lumbar interbody fusion without decompression for the treatment of symptomatic degenerative spondylolisthesis at L4-5. Neurosurg Focus. 2018;44(1):E6. doi:10.3171/2017.10.FOCUS17566

61. Hijji FY, Narain AS, Bohl DD, et al. Lateral lumbar interbody fusion: a systematic review of complication rates. Spine J. 2017;17(10):1412-1419. doi:10.1016/j.spinee.2017.04. 022

62. Lehmen JA, Gerber EJ. MIS lateral spine surgery: a systematic literature review of complications, outcomes, and economics. Eur Spine J. 2015;24(suppl 3):287-313. doi:10.1007/ s00586-015-3886-1

Disclosures and COI: The authors received no funding for this study and report no conflicts of interest.

Corresponding Author: Kern Singh, MD, Professor, Department of Orthopaedic Surgery, Rush University Medical Center, 1611 West Harrison Street, Suite 300, Chicago, IL 60612. Phone: (312) 432-2373; Fax: (708) 409-5179; Email: kern. singh@rushortho.com.

Published 22 January 2021

This manuscript is generously published free of charge by ISASS, the International Society for the Advancement of Spine Surgery. Copyright (C) 2020 ISASS. To see more or order reprints or permissions, see http://ijssurgery.com.

\section{APPENDIX 1. MULTIMODAL ANALGESIC REGIMEN FOR OUTPATIENT SPINE SURGERY}

\section{Prior to Admission}

Preoperative patient counseling regarding intraoperative and postoperative analgesia at spine surgeon's office.

\section{Day of Surgery}

\section{Preoperatively}

Oral medications given preoperatively in holding area about 1 hour prior to surgery:

1. Cyclobenzaprine $10 \mathrm{mg}$
2. Pregabalin $150 \mathrm{mg}$

3. Oxycodone controlled release $10 \mathrm{mg}$

\section{Intraoperatively}

Induction of anesthesia-propofol $2 \mathrm{mg} / \mathrm{kg}$ plus ketamine $50 \mathrm{mg}$

Maintenance of anesthesia-sevoflurane with fentanyl $1-2 \mu \mathrm{g} / \mathrm{kg}$ titrated to clinical effect

Additional medications administered intraoperatively

1. Bupivacaine $0.5 \%$ with epinephrine $1: 200000$ injected at incision site

a. $20 \mathrm{~mL}$ per side if patient weight $<70 \mathrm{~kg}$

b. $30 \mathrm{~mL}$ per side if patient weight $>70 \mathrm{~kg}$

2. Acetaminophen $1000 \mathrm{mg} \mathrm{IV}$

3. Dexamethasone $10 \mathrm{mg} \mathrm{IV}$

4. Ondansetron $4 \mathrm{mg} \mathrm{IV}$

\section{Postoperatively in Recovery Room}

1. Tramadol $50 \mathrm{mg}$

2. Cyclobenzaprine $10 \mathrm{mg}$ orally for spasms

3. Oxycodone immediate release

a. $5 \mathrm{mg} \mathrm{q} 4 \mathrm{~h}$ as needed for pain (VAS $>3$ ) for opioid-naive patients

b. $10 \mathrm{mg} \mathrm{q} 4 \mathrm{~h}$ as need for pain (VAS $>4$ ) for opioid-tolerant patients

\section{Discharge Medications}

Postoperative day 0

1. Tramadol $50 \mathrm{mg}$

2. Oxycodone $5 \mathrm{mg}$

a. $5 \mathrm{mg}$ as needed for pain (VAS 4-6)

b. $10 \mathrm{mg}$ as needed for pain (VAS 7-10)

3. Cyclobenzaprine $10 \mathrm{mg}$

4. Pregabalin $75 \mathrm{mg}$

5. Cold compress applied to surgical site

\section{Postoperative day 1}

1. Oxycodone discontinued by 9 am

2. Hydrocodone/paracetamol $5 \mathrm{mg}$

a. 1 tablet as needed for pain (VAS 4-6)

b. 2 tablets as needed for pain (VAS 7-10)

3. Cyclobenzaprine $10 \mathrm{mg}$

Abbreviation: VAS $=$ visual analog scale for pain (where $0=$ no pain and $10=$ worst possible pain) 


\section{APPENDIX 2. PATIENT SELECTION}

Table A1. Recommendations for patient selection for lumbar fusion in ambulatory surgery center (ASC) settings.

Patient factors that may exclude patients from ASC

Age $>65 \mathrm{y}^{2,25}$

BMI $>40 \mathrm{~kg} / \mathrm{m}^{2}(13)$

Increased risk of postoperative nausea and vomiting ${ }^{7}$

Patient does not have a reliable caregiver at home ${ }^{20}$

Patient does not have functional independence and cannot perform activities of daily living by himself/herself $f^{2,25}$

Medical comorbidities (e.g., congestive heart failure, myocardial infarction within 6 mo, angina pectoris, ASA score $\geq 3$, increased risk of thromboembolism, obstructive sleep apnea $)^{20}$

Surgical factors that may exclude patients from ASC

Increased operative duration (greater than $2 \mathrm{~h}$ )

Increased surgical invasiveness

Spinal deformity 4 